\title{
OCEAN ACOUSTIC TOMOGRAPHY
}

\author{
The sense of excitement and discovery remains.
}

\author{
By Walter H. Munk and Peter F. Worcester \\ Scripps Institution of Oceanography. University of California at San Diego, La Jolla. CA 92093
}

A natural

question to ask

was whether

acoustics could

be made the

basis of an ocean

mapping scheme.

The specific

proposal was

to use acoustic

travel times

between a set

of moorings.
$\mathrm{I}_{\mathrm{n}}$ the early 1960 's, the physical oceanography community was rudely awakened from their pursuit of "Direct Current"(D.C.) oceanography. John Swallow had acoustically tracked deep floats; instead of drifting in parallel at a few $\mathrm{mm}$ per second. as predicted by circulation models, the floats moved in different directions at $10 \mathrm{~cm} / \mathrm{s}$. There followed a series of ambitious experiments: the Soviet POLYGON moorings, the Mid-Ocean Dynamics Experiment (MODE) and POLYMODE. By the mid-1970's it had become clear that most of the pelagic kinetic energy is associated not with the steady circulation but with eddies of $100 \mathrm{~km}$ and 100 day scales.

The mesoscale eddies posed a new challenge to ocean sampling. It takes a vessel roughly six weeks to map a square megameter $(1 \mathrm{Mm}=1000$ $\mathrm{km}$ ) with mesoscale resolution using CTDs, and by then things have changed. Speeding up the research vessels does not look promising; it was 10 knots in Challenger's time and it is 10 knots today.

Sound travels at 3000 knots (about $1500 \mathrm{~m} / \mathrm{s}$ ), and is effectively transmitted through the oceans (in 1960 an explosion off Perth, Australia was recorded by Bermuda hydrophones at $20,000 \mathrm{~km}$ range). A natural question to ask was whether acoustics could be made the basis of an ocean mapping scheme. The specific proposal was to use acoustic travel times between a set of moorings. There are $0.5 \mathrm{~N}(\mathrm{~N}-1)$ paths between $\mathrm{N}$ moorings, and so the data return increases quadratically with $N$ (and it also decreases quadratically with mooring failure, as we later painfully learned). The ocean sound channel (SOFAR channel) acts as a wave guide, with separate arrivals from the early steep rays which oscillate through the entire ocean column, to the late flat rays which remain near the sound axis at $1 \mathrm{~km}$. Six moorings with 10 multipaths per sourcereceiver pair yield a data set of 150 travel times per transmission. It is the task of inverse theory to invert this data set into optimum maps of the sound speed field $C(x, y, z)$ which in turn is closely related to the temperature field $T(x, y, z)$. According to Radon's theorem. the mapping can be achieved with arbitrarily small error by going to an arbitrarily large number of rays, but this does not help much. The question is whether an affordable array can yield useful maps.

There is of course nothing new in using acoustic travel times to derive information about the medium. Seismology is based on this applica- tion. But for the solid earth it may be the only way to get information about an inaccessible interior. For the oceans there is a choice. Could it be that the availability of traditional "contact measurements" has been a liability by discouraging the development of more effective methods of remote sensing?

We must refer the reader to published material for details on the experimental procedure and on the inverse processing (Cornuelle, et al., 1985; Howe, et al., 1987; Munk and Wunsch, 1979, 1982; Cornuelle and Howe, 1987). The procedure requires the resolution of separate ray arrivals, the identification of an arrival pulse with a known ray path, and the stability of acoustic ray paths for tracking the ray travel times for the duration of the experiment. A 1979 experiment established this feasibility. (This was crucial since a reviewer had claimed that resolution, identification, and stability of ray paths were contrary to physical law.) A 1981 experiment in the MODE area (Cornuelle, et al., 1985) southwest of Bermuda demonstrated the ability to perform somewhere between crude mapping and pattern recognition (Fig. 1). Four source moorings and 5 receiver moorings were deployed on the periphery of a $300 \mathrm{~km}$ square. We used a Webb source at $224 \mathrm{~Hz}$ center frequency with an effective bandwidth df of about $10 \mathrm{~Hz}$. The resolution is accordingly $(\mathrm{df})^{-1}=100 \mathrm{~ms}$.

In retrospect we can identify two basic weaknesses with the 1981 deployment (which we had billed as a "demonstration"). The bandwidth (and hence resolution) was inadequate for a clean separation of successive ray arrivals. This presented a "Catch 22" situation: we needed the resolution of broad-band sources to demonstrate the feasibility, and we needed to demonstrate the feasibility to gain support for acquiring the broad band sources! We barely squeaked by; since 1983 sources with $\mathrm{df}=100 \mathrm{~Hz}$ bandwidth have given satisfactory resolution (Fig. 2).

The second weakness was that the deployed array was too sparse for good mapping. But we shall never be in a position of deploying many more moorings. Accordingly we are now developing a strategy which combines a small number of source moorings with a movable ship-based receiver. Computer realizations indicate the feasibility of producing maps over a $\mathrm{Mm}$ square that can account for $95 \%$ of the mesoscale variance. Sea trials are starting in late 1988.

We are well aware that mapping is only a step 


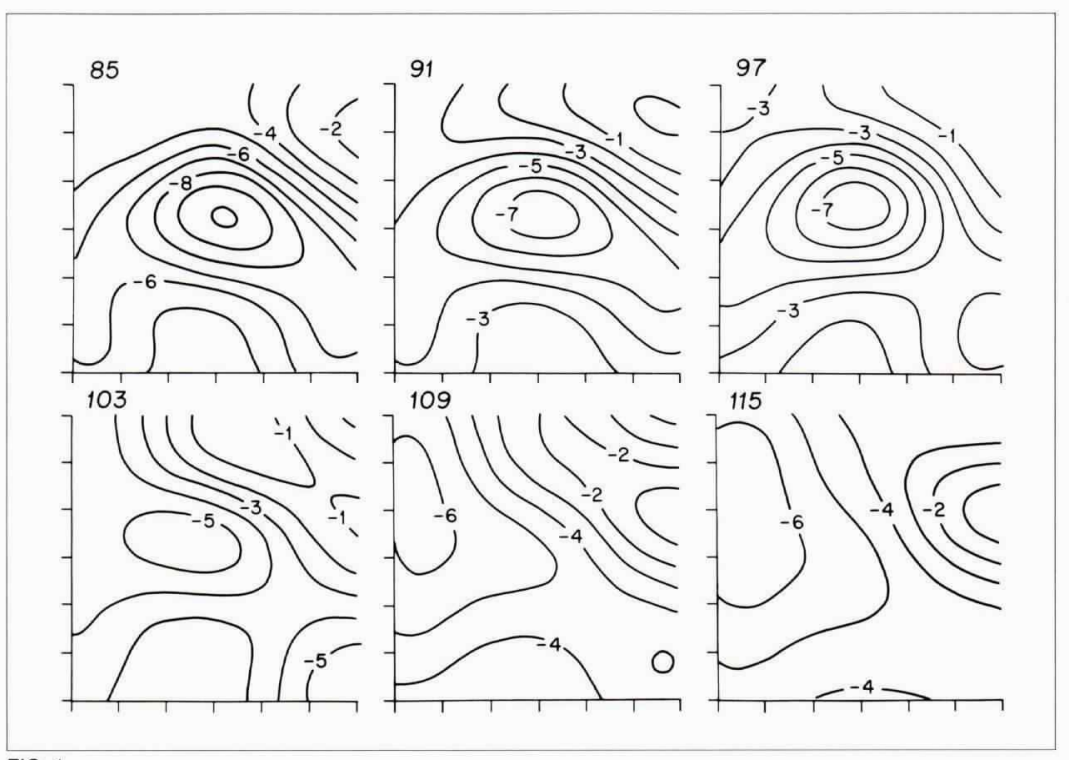

FIG. 1

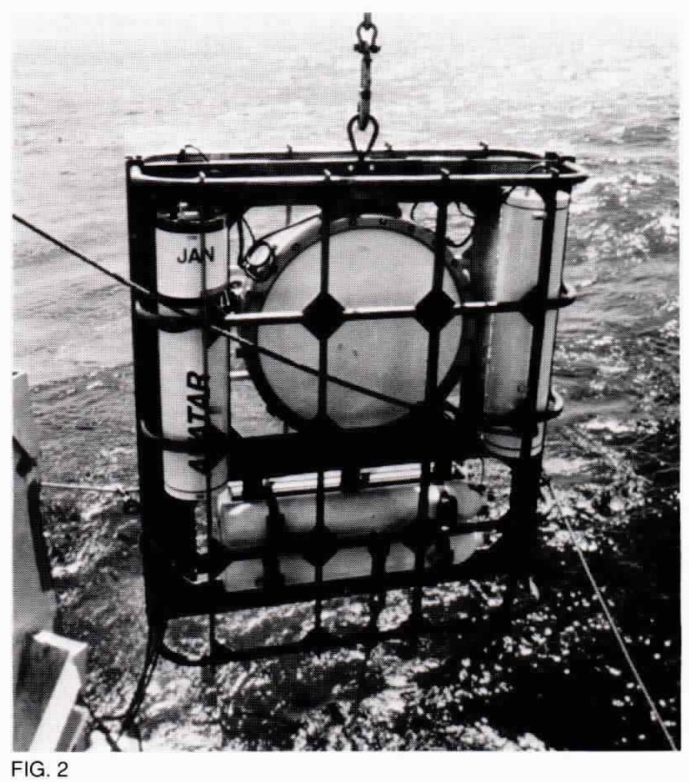

Fig. 1. The acoustic travel time data obtained in the 1981 Ocean Acoustic Tomography Experiment was used to draw maps of the ocean sound speed field at $700 \mathrm{~m}$ depth in the $300 \mathrm{~km}$ by $300 \mathrm{~km}$ area of the experiment. Maps at six-day intervals from year day 85 through 115 show a cold eddy with low sound speed remaining stationary for several days and then moving rapidly out of the experiment area beginning on year day 103. The sound speed contours relative to $1506 \mathrm{~m} / \mathrm{s}$ are drawn at intervals of $1 \mathrm{~m} / \mathrm{s}$, which roughly corresponds to a temperature change of 0.2 degrees Celsius. Maps at other depths can also be constructed using the acoustic data.

Fig. 2. Tomography transceiver used in the 1986/87 Gyre-Scale Reciprocal Transmission Experiment. This is a second generation transceiver. using an HLF-5 acoustic source from Hydroacoustics Inc. and a newly designed set of electronics. The high pressure nitrogen bottles in the bot-

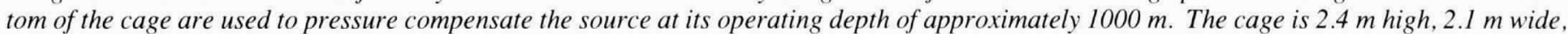
and about $1 \mathrm{~m}$ thick; it weighs roughly $1590 \mathrm{~kg}$ in air and $590 \mathrm{~kg}$ in water.

towards the recognition and understanding of ocean processes, such as the apportionment of energy between barotropic and baroclinic waves, the distribution of energy among the baroclinic modes, the departure from geostrophy, the degree of mode coupling, etc. The beauty of the inverse method (whether applied to tomography or elsewhere) is that it is readily adapted to ask these questions directly from the data (without necessarily going through the intermediary mapping stage), and to design the experiments so that the answers are found with an adequate (and known) probability level.

So far we have emphasized the resolution limits imposed by source bandwidth. Another consideration is the ratio between the mesoscale-induced travel time fluctuations (the signal) and the high frequency jitter in travel times (the noise). The latter is predominantly associated with internal wave activity. (Acoustic noise-induced travel time fluctuations play a negligible role in these acoustic experiments.) A typical value for the signal-to-noise ratio of the variances at $1 \mathrm{Mm}$ range is $(200 \mathrm{~ms})^{2} /(10 \mathrm{~ms})^{2}=400$.

Co-located sources and receivers can be used to form the travel time differences, A-to-B minus Bto- $\mathrm{A}$, as a measure of the component of ocean current along the transmission path (Fig. 3). For mesoscale eddies the velocity signal is an order of magnitude weaker than the temperature signal, $20 \mathrm{~ms}$ rather than $200 \mathrm{~ms}$, suggesting an unsatisfactory $\mathrm{S} / \mathrm{N}$ ratio of $4: 1$. The noise is the result of temperature fluctuations induced along the path by internal wave activity. But if the oppositelydirected paths were truly reciprocal they would see the same temperature structure, and so the effect cancels in the travel time difference.
However, the paths cannot be truly reciprocal, due to refraction by current shear, non-simultaneity, etc. What is the degree of cancellation? It was found that the reciprocal noise variance at 300 $\mathrm{km}$ range is in fact smaller than the one-way noise variance by a factor of 10 , so that $\mathrm{S} / \mathrm{N}$ is happily $40: 1$ rather than $4: 1$ : thus velocity tomography is feasible. By measuring differences along a variety of paths the inversion procedure leads to the vector velocity, $\mathbf{u}(x, y, z)$. The simultaneous application of temperature tomography and velocity tomography provides some interesting possibilities, such as the comparison of barotropic and baroclinic energies and a test of the thermal wind relation.

We have recently completed a reciprocal transmission experiment around three moorings in a $1000 \mathrm{~km}$ triangle north of Hawaii. The circulation about the periphery is found from a singaround, travel time A-to-B-to-C-to-A minus Ato-C-to-B-to-A. Stokes' theorem yields the mean relative vorticity, and separate analysis of steep and flat rays yield relative vorticity as a function of depth. The purpose is to derive the vorticity response to wind-torquing. This is a gyre dynamics experiment, with no provisions for mesoscale mapping. It exploits the capability of long-range acoustic transmissions to integrate over long distances and so suppress the small scale processes.

We are now preparing for a reciprocal transmission experiment in the Greenland Sea, with five moorings at the corners of a pentagon and one in the center. Following deployment and preceding recovery we are planning to occupy receiving stations around the periphery as initial tests of moving ship tomography (Fig. 4). But the em-
The beauty

of the inverse

method is that

it is readily

adapted to ask

these questions

directly from

the data, and

to design the

experiments so

that the answers

are found with

an adequate

probability level. 


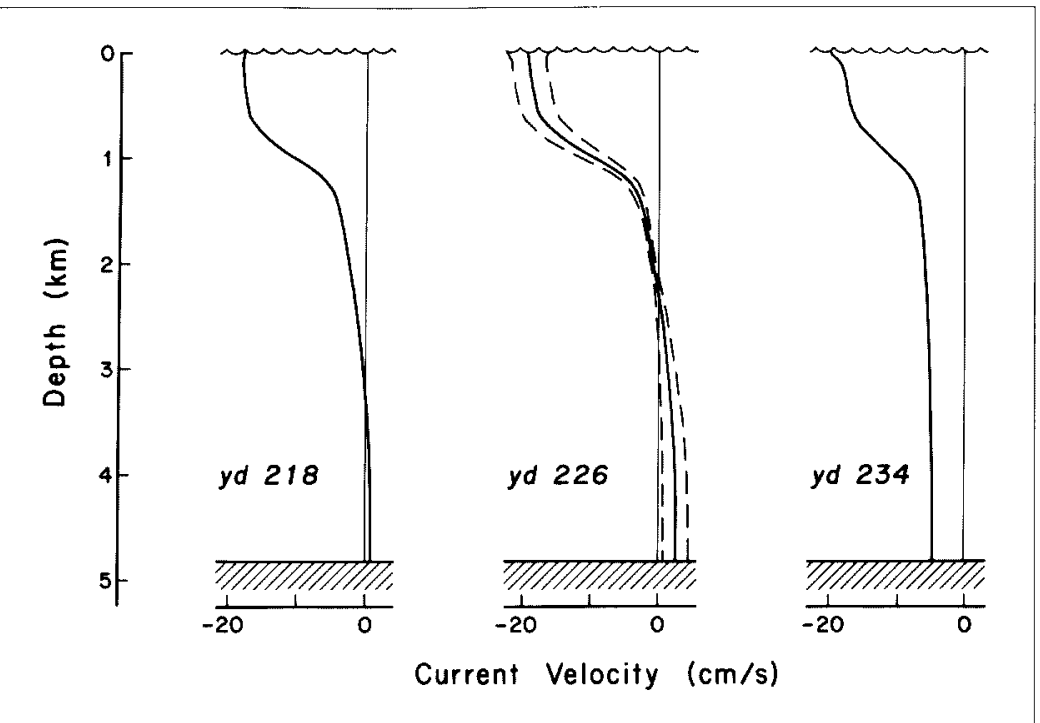

FIG. 3

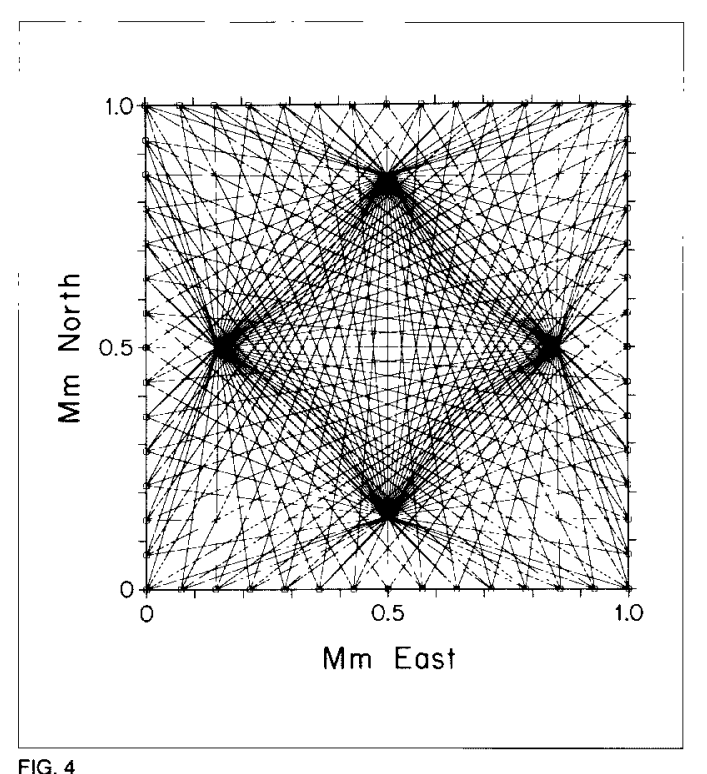

FIG. 4

Fig. 3. The differences in travel times between sound pulses traveling in opposite directions in the 1983 Reciprocal Acoustic Transmission Experiment were used to compute the average current velocity in the $300-\mathrm{km}$ long vertical slice between the transceiver moorings as a function of depth. Three profiles obtained at eight day intervals show the slow changes that occur in the current profiles in the ocean. All have stronger currents near the surface than at great depths, as is typical of ocean currents. The calculated errors in the profiles are approximately 1 cmis, as shown by the dashed lines around the central profile. Note the change from a baroclinic mode structure on year day 218 to a strong barotropic (top-to-bottom) component on year day 234.

Fig. 4. Plan view of the ray geometry in a simulated moving ship tomography experiment. Four sources are located on a 350-km radius circle about the center of a $1000 \mathrm{~km}$ by $1000 \mathrm{~km}$ square. Receivers are located at approximately $70 \mathrm{~km}$ intervals around the square. In this simulation the ocean is assumed to be 2-dimensional, with no vertical structure; straight line ray paths can therefore be used in the calculation. The inversion procedure (using realistic travel time errors) leads to mapping which accounts for $95 \%$ of the mesoscale variance. Subsequent 3-D simulations with vertical multipaths lead to similar mapping errors. In this preliminary simulation all receptions are assumed simultaneous. The actual experiment will be conducted by lowering a receiver from a ship at roughly $70 \mathrm{~km}$ intervals as it transits around the square. This involves " $n o w$ casting" in a 4-D (space + time) environment.

We wish we

could enumerate

the great things

we have done for

oceanography;

in fact till now

we have learned

more about

acoustics

than about

oceanography. phasis is on vorticity forcing in high latitudes and on convective overturn during winter cooling. The moorings will go in during September 1988 and come out a year later.

Tomography primarily measures temperature, and is insensitive to salinity, which is of critical importance in the dynamics of Arctic regions. Accordingly the acoustic measurements are embedded in a program involving CTD surveys, current meter moorings, satellite altimetry, etc. The inversion procedure is well adapted to accepting a disparate data set, allowing for the qualitative differences in precision and sampling rate. In the case of the Greenland Sea Experiment, the tomographic sampling at 4 hourly intervals plays an important role in interpolating sparse data. Is the convective overturn and the formation of deep water gradual or catastrophic?

Some, perhaps all, of the array will be covered by ice during the winter months. The arctic sound channel is a surface duct, with refracted/surfacereflected $(\mathrm{R} / \mathrm{SR})$ transmission and so is sensitive to surface boundary conditions. Does the progressive ice cover lead to some unsuspected transition phenomena? There are opportunities for learning where we don't even know what the questions are. There is an element of adventure, and we welcome it.

And so ends this brief essay of 10 years spent in the development and application of a new tool in the exploration of the sea. The sense of excitement and discovery remains. We wish we could enumerate the great things we have done for oceanography; in fact, until now we have learned more about acoustics than about oceanography.

The work has been very much of a collaborative effort. R. Spindel of Woods Hole (now at University of Washington, with $\mathrm{B}$. Howe) oversaw the mooring technology and position keeping. C. Wunsch and B. Cornuelle (MIT) adapted inverse theory to the tomographic problem. T. Birdsall and K. Metzger of the University of Michigan are responsible for the innovative coding and signal processing procedures (and they pulled numerous dB's out of the hat). There are numerous others. We have worked together by Telemail and shared enthusiasm, without a professional coordinator. At one stage a reviewer termed our organizational structure a disaster, but gave the proposal his reluctant support when we pointed to forty published papers. We are deeply indebted to our colleagues in ONR and NSF for their participation and support.

\section{REFERENCES}

Cornuelle, Bruce, et al. (1985). Tomographic maps of the ocean mesoscale. Part 1: Pure acoustics. I. Phys. Oceanogr., 15. $133-152$.

Cornuelle. Bruce and Bruce M. Howe (1987). High spatial resolution in vertical slice ocean acoustic tomography, $J$. Geophys. Res., 92, 11,680-11,692.

Howe, Bruce M.. Peter F. Worcester and Robert C. Spindel (1987). Ocean acoustic tomography: Mesoscale velocity. $J$. Geophys. Res. 92, 3785-3805.

Geophys. Res., 92, 3785-3805.
Munk, Walter H. and Carl Wunsch (1979). Ocean acoustic tomography: a scheme for large scale monitoring. Deep-Sea Res., 26A, 123-161.

Munk. Walter H. and Carl Wunsch (1982). Observing the ocean in the 1990 s. Phil. Trans. Roy. Soc. London, 307.439464. 